\title{
Optimal investment and premium control in a nonlinear diffusion model
}

\author{
Ming Zhou * \\ China Institute for Actuarial Science \\ Central University of Finance and Economics \\ 39 South College Road, Haidian, Beijing 100081, China \\ mzhou.act@gmail.com \\ Kam C. Yuen \\ Department of Statistics and Actuarial Science \\ The University of Hong Kong \\ Pokfulam, Hong Kong, China \\ kcyuen@hku.hk \\ Chuancun Yin \\ School of Mathematical Sciences \\ Qufu Normal University \\ Shandong 273165, China \\ ccyin@mail.qfnu.edu.cn
}

*Corresponding author. 


\begin{abstract}
This paper considers the optimal investment and premium control problem in a diffusion approximation to a non-homogeneous compound Poisson process. In the nonlinear diffusion model, it is assumed that there is an unspecified monotone function describing the relationship between the safety loading of premium and the time-varying claim arrival rate. Hence, in addition to the investment control, the premium rate can be served as a control variable in the optimization problem. Specifically, the problem is investigated in two cases: (i) maximizing the expected utility of terminal wealth, and (ii) minimizing the probability of ruin respectively. In both cases, some properties of the value functions are derived, and closed-form expressions for the optimal policies and the value functions are obtained. The results show that the optimal investment policy and the optimal premium control policy are dependent on each other. Most interestingly, as an example, we show that the nonlinear diffusion model reduces to a diffusion model with a quadratic drift coefficient when the function associated with the premium rate and the claim arrival rate takes a special form. This example shows that the model of study represents a class of nonlinear stochastic control risk model.
\end{abstract}

Keywords CARA utility, Dependent control policies, Hamilton-Jacobi-Bellman equation, Investment, Premium control. 


\section{Introduction}

In the past few decades, ruin probability and other related actuarial variables are mainly treated in risk theory and mathematical insurance. However, some optimization problems are becoming more and more interesting in recent years. From the viewpoint of insurers, optimal dividend, investment and/or reinsurance problems are extensively studied. For example, see Schmidli (2008). In this paper, we formulate a dynamic risk model in which an insurer can control premium loading and investment strategy to achieve utility maximization and risk minimization.

In the actuarial literature, the surplus process $\left\{Y_{t}, t \geq 0\right\}$ of an insurance portfolio is usually described by the classical Cramér-Lundberg model

$$
Y_{t}=x+C_{t}-\sum_{k=1}^{N_{t}} X_{k}
$$

where $\left\{N_{t}, t \geq 0\right\}$ is a counting process describing the total number of claims up to time $t$; the claim sizes $\left\{X_{k}, k=1,2, \cdots\right\}$, independent of $\left\{N_{t}, t \geq 0\right\}$, are positive independent and identically distributed (i.i.d) random variables with finite first and second moments, $\mu$ and $\sigma^{2}$, respectively; and $C_{t}$ is the accumulated premium income prior to time $t$.

In the classical risk model, it is usually assumed that $\left\{N_{t}, t \geq 0\right\}$ is a homogeneous Poisson process, which implies that the claims arrive at a constant rate. However, the arrival rate of claims often varies with time in practice. Intuitively, the claim arrival rate of an insurance portfolio depends on the portfolio size which in turn relies heavily on the premium rate charged by the insurance company. As one can observe in the insurance market, an insurance company can take advantage of adjusting the premium rate to achieve certain target. Once the premium rate is changed, the size of the insurance portfolio as well as the claim arrival rate will change. This fact suggests that there exists a monotone function mapping between the premium rate and the claim arrival rate. In view of this, we consider a relationship between the two rates in the present paper. As a result, the premium rate or the claim arrival rate can be served as a control variable in our optimization problem. In fact, not much research in this direction has been done in the past few decades. For related work, see Martin-Löf (1983), and Vandebroeka and Dhaenea (1990). Recently, Højgaard 
(2002) studies the optimal dividend problem in the compound Poisson model by taking an unspecified function between the safety loading of premium and the claim arrival rate; and Asmussen et al. (2013) consider the portfolio size as a function of premium, and derives the optimal premium by minimizing ruin probability.

The purpose of this paper is to study the optimal investment and premium control problem in a diffusion approximation to a non-homogeneous compound Poisson process with time-varying intensity. In particular, similar to the work of Højgaard (2002), we use an unspecified monotone function to describe the relationship between the safety loading of premium and the claim arrival rate. Under the diffusion model, we consider the optimization problem of maximizing the expected utility of terminal wealth and that of minimizing the ruin probability. In both cases, it can be shown that the optimal investment policy and the optimal premium control policy are dependent on each other. Most interestingly, in a special case, the diffusion model of study reduces to a nonlinear diffusion model with a quadratic drift coefficient.

The rest of the paper is organized as follows. In Section 2, we describe how the diffusion model of study is derived from a non-homogeneous compound Poisson process. In Section 3 , we derive the optimal investment and premium control policies and the value functions for the two optimization objectives. Finally, in Section 4, we connect the safety loading of premium and the claim arrival rate through a specific function, under which explicit optimal investment and premium control policies can be obtained.

\section{The model}

In model (1.1), we assume that $\left\{N_{t}, t \geq 0\right\}$ is a non-homogeneous Poisson process with time-varying deterministic rates $\lambda(s)$, and that the premium is calculated by the expected value principle with a time-varying relative safety loading $\theta_{t}$. Then, we have

$$
C_{t}=\mu \int_{0}^{t}\left(1+\theta_{s}\right) \lambda(s) d s .
$$

In this set-up, the insurer can adjust the premium rate dynamically, and the safety loading $\theta$ being the only parameter in the premium rate turns out to be a control variable. As was 
mentioned before, when the level of the safety loading rises, the portfolio size as well as the claim arrival rate drop. Hence, it is reasonable to express the claim arrival rate as a strictly decreasing function of the safety loading, say $\lambda_{t}=h\left(\theta_{t}\right)$ with $h(\infty)=0$. Furthermore, it is natural to assume that there exists a finite number of potential customers in the market so that $h(0)=\lambda_{\max }<\infty$. As a consequence, we have

$$
\begin{aligned}
& \mathbb{E}\left[d Y_{t}\right]=d C_{t}-\mathbb{E}\left[d \sum_{k=1}^{N_{t}} X_{k}\right]=\theta_{t} h\left(\theta_{t}\right) \mu d t, \\
& \mathbb{D}\left[d Y_{t}\right]=\mathbb{D}\left[d \sum_{k=1}^{N_{t}} X_{k}\right]=h\left(\theta_{t}\right) \sigma^{2} d t,
\end{aligned}
$$

where $\mathbb{E}$ and $\mathbb{D}$ denote the expectation operator and variance operator respectively. Because of (2.2) and (2.3), we further assume that $\theta h(\theta) \rightarrow 0$ as $\theta \rightarrow \infty$. This assumption ensures that the insurer cannot make a profit by charging a positive premium rate without taking any risk.

Suppose that the relationship between the safety loading $\theta$ and the claim arrival rate $\lambda$ is given by $\lambda=h(\theta)$, and that the function $h$ satisfies the following assumptions.

\section{Assumption A:}

1. $h$ is strictly decreasing on $[0, \infty)$ with $h(0)=\lambda_{\max }$ and $h(\infty)=0$;

2. $h(x) x \rightarrow 0$ as $x \rightarrow \infty$.

Since $h$ is a strictly decreasing function, we take the claim arrival rate $\lambda$, instead of $\theta$, as a control variable. Denote the inverse function of $h$ by $h^{-1}$. Then, the surplus process of the insurance portfolio can be rewritten as

$$
d Y_{t}^{\lambda}=\left(1+h^{-1}\left(\lambda_{t}\right)\right) \lambda_{t} \mu d t-d \sum_{k=1}^{N_{t}^{\lambda}} X_{k}, \quad X_{0}^{\lambda}=x
$$

where $\left\{N_{t}^{\lambda}, t \geq 0\right\}$ is a non-homogeneous Poisson process with time-varying intensity $\left\{\lambda_{t}, t \geq\right.$ $0\}$.

We now present a diffusion approximation to the non-homogeneous compound Poisson process (2.4). Let $\left\{N^{1}(t), t \geq 0\right\}$ be a homogeneous Poisson process with a unit rate. Then, 
it follows from Jabari and Liu (2013) that

$$
\sqrt{n}\left(\frac{1}{n} N^{1}(n t)-t\right) \approx \frac{1}{\sqrt{n}} \sum_{k=1}^{\lfloor n t\rfloor}\left(N^{1}(k)-N^{1}(k-1)-1\right) \stackrel{\mathcal{D}}{\approx} W(t),
$$

where $\lfloor n t\rfloor$ is the integral part of $n t$, and $\{W(t), t \geq 0\}$ is a standard Brownian motion. By letting the scaled time $\tilde{t}=n t$, we get

$$
N^{1}(\tilde{t})-\tilde{t} \stackrel{\mathcal{D}}{\approx} \sqrt{n} W\left(\frac{\tilde{t}}{n}\right) \stackrel{\mathcal{D}}{=} W(\tilde{t})
$$

where $\stackrel{\mathcal{D}}{\approx}$ means "approximately equal, in distribution, to" and $\stackrel{\mathcal{D}}{=}$ denotes equivalence in distribution. Thus, for the non-homogeneous Poisson process $\left\{N^{\lambda}(t), t \geq 0\right\}$, we have

$$
N_{t}^{\lambda} \stackrel{\mathcal{D}}{=} N^{1}\left(\int_{0}^{t} \lambda_{s} d s\right)
$$

and

$$
N_{t}^{\lambda}-\int_{0}^{t} \lambda_{s} d s \stackrel{\mathcal{D}}{\approx} W\left(\int_{0}^{t} \lambda_{s} d s\right) \stackrel{\mathcal{D}}{=} \int_{0}^{t} \sqrt{\lambda_{s}} d W_{s}
$$

Along the same lines, for the non-homogeneous compound Poisson process $\left\{\sum_{k=1}^{N_{t}^{\lambda}} X_{k}, t \geq 0\right\}$, we obtain

$$
\sum_{k=1}^{N_{t}^{\lambda}} X_{k}-\mu \int_{0}^{t} \lambda_{s} d s \stackrel{\mathcal{D}}{\approx} \sigma W\left(\int_{0}^{t} \lambda_{s} d s\right) \stackrel{\mathcal{D}}{=} \sigma \int_{0}^{t} \sqrt{\lambda_{s}} d W_{s} .
$$

Thus, the diffusion approximation process $\left\{Y_{t}^{\lambda}, t \geq 0\right\}$ to (2.4) can be written as

$$
d Y_{t}^{\lambda}=\mu h^{-1}\left(\lambda_{t}\right) \lambda_{t} d t+\sigma \sqrt{\lambda_{t}} d W_{t}^{Y}, \quad Y_{0}^{\lambda}=x
$$

where $\left\{W_{t}^{Y}, t \geq 0\right\}$ is a standard Brownian motion.

For mathematical convenience, we make a change of variable by setting $u=\sqrt{\lambda}$ and use $u$ as a control variable. Assume that there exists a fixed cost rate (or debit rate) $c>0$ for the insurance portfolio. Then, the surplus at time $t$ satisfies the Itô stochastic differential equation

$$
d Y_{t}^{\lambda}=\left[\mu G\left(u_{t}\right)-c\right] d t+\sigma u_{t} d W_{t}^{Y}, \quad Y_{0}^{\lambda}=x
$$

where

$$
G(u)=u^{2} h^{-1}\left(u^{2}\right)
$$

From Assumption A, we can derive some properties of the function $G$ in the following lemma. 
Lemma 2.1. The function $G:\left[0, \sqrt{\lambda}_{\max }\right] \rightarrow[0, \infty)$ satisfies

$$
G(0)=G\left(\sqrt{\lambda}_{\max }\right)=0, \quad x G^{\prime}(x) \rightarrow 0, \quad \text { and } \quad \frac{x}{G^{\prime}(x)} \rightarrow 0, \quad \text { as } \quad x \rightarrow 0+.
$$

Proof. It follows from Assumption A that $h(0)=\lambda_{\max }, h(\infty)=0$, and $x h(x) \rightarrow 0$ as $x \rightarrow \infty$. Since $h^{-1}$ is the inverse function of $h$, we have $h^{-1}\left(\lambda_{\max }\right)=0$ and $h^{-1}(0)=\infty$. In addition,

$$
x h^{-1}(x)=h\left(h^{-1}(x)\right) h^{-1}(x) \rightarrow 0, \quad \text { as } \quad x \rightarrow 0,
$$

simply because $h^{-1}(x) \rightarrow \infty$ as $x \rightarrow 0$ and $x h(x) \rightarrow 0$ as $x \rightarrow \infty$. Thus, we have $G(0)=$ $G\left(\sqrt{\lambda}_{\text {max }}\right)=0$. It also suggests that, as $x \rightarrow 0+$,

$$
h^{-1}(x) \sim x^{-\epsilon}, \quad 0<\epsilon<1
$$

and hence

$$
G^{\prime}(x)=2 x h^{-1}\left(x^{2}\right)+2 x^{3} h^{-1^{\prime}}\left(x^{2}\right) \sim x^{-2 \epsilon+1},
$$

and

$$
x G^{\prime}(x) \sim x^{-2 \epsilon+2}, \quad \frac{x}{G^{\prime}(x)} \sim x^{2 \epsilon}, \quad \text { as } \quad x \rightarrow 0 .
$$

By noting that $-2 \epsilon+2$ and $2 \epsilon$ are positive, we complete the proof of the lemma.

To end the section, we state another two assumptions which are useful in deriving the main results of the paper.

Assumption B: $G(x)$ is a strictly concave function on $\left[0, \sqrt{\lambda}_{\max }\right]$.

Note that the function $G$ is strictly concave with $G(0)=G\left(\sqrt{\lambda}_{\text {max }}\right)=0$. There exists a unique point in $\left[0, \sqrt{\lambda}_{\max }\right]$ at which $G$ attains its maximum. If $\bar{u}$ is the point, i.e., $G^{\prime}(\bar{u})=0$, then $G$ is strictly increasing on $[0, \bar{u}]$ and strictly decreasing for $u>\bar{u}$. So, for $u>\bar{u}$, the volatility coefficient of the insurance portfolio is increasing but the drift coefficient is decreasing (see (2.5)). Since this is unacceptable for a risk-averse insurer, we only focus on the control variable $u \in[0, \bar{u}]$. Moreover, in order to have a positive drift coefficient in (2.5), we need the following assumption.

Assumption C: $\mu G(\bar{u})-c>0$, i.e., $G(\bar{u})>c / \mu$. 


\section{Optimizations with investment and premium control}

Besides the insurance risk, we assume that the insurer also faces a classical Black-Sholes financial market, in which there are one risky asset (e.g., a mutual fund) and one risk-free asset. The risky asset's price process $\left\{S_{t}, t \geq 0\right\}$ is assumed to follow a geometric Brownian motion, i.e., $S_{t}$ satisfies the Itô stochastic differential equation

$$
d S_{t}=\alpha S_{t} d t+\beta S_{t} d W_{t}^{S}
$$

where $\alpha$ and $\beta$ are positive constants, and $\left\{W_{t}^{S}, t \geq 0\right\}$ is a standard Brownian motion. As usual, it is assumed that $\left\{W_{t}^{Y}, t \geq 0\right\}$ and $\left\{W_{t}^{S}, t \geq 0\right\}$ are independent, and that the risk-free asset has a fixed continuously compound return rate $r \geq 0$. To avoid triviality, we put $\alpha \geq r$.

Suppose that the insurer invests an amount of $\pi_{t}$ in the risky asset at time $t$, and that the remaining surplus of the insurance portfolio accumulates at the compound interest rate $r$. Then, with an investment policy $\pi$ and a premium control policy $u$, the surplus process of the insurance portfolio can be expressed as

$$
d Y_{t}^{\pi, u}=\left(r Y_{t}^{\pi, u}+\mu G\left(u_{t}\right)+\pi(\alpha-r)-c\right) d t+\sigma u_{t} d W_{t}^{Y}+\beta \pi_{t} d W_{t}^{S}, \quad Y_{0}^{\pi, u}=x .
$$

For a pair of Markov control processes $(\pi, u)$, and any function $f(t, x) \in \mathcal{C}^{1,2}$, we define an operator $\mathcal{A}^{\pi, u}$ associated with the surplus process (3.1) as

$$
\mathcal{A}^{\pi, u} f(t, x)=f_{t}+\frac{1}{2}\left[\sigma^{2} u_{t}^{2}+\beta^{2} \pi_{t}^{2}\right] f_{x x}+\left[r x-c+\mu G\left(u_{t}\right)+\pi_{t}(\alpha-r)\right] f_{x} .
$$

Furthermore, we denote the set of all admissible policies by $\mathcal{Z}$. We say that a pair of policy $(\pi, u) \in \mathcal{Z}$ is admissible if

1. The process $u=\left\{u_{t}, t \geq 0\right\}$ is a predictable such that $0 \leq u_{t} \leq \bar{u}$;

2. The process $\pi=\left\{\pi_{t}, t \geq 0\right\}$ is a predictable process such that

$$
\mathbb{E} \int_{0}^{T} \pi_{s}^{2} d s<\infty, \quad \forall T<\infty ;
$$

3. The stochastic differential equation (3.1) determines a unique strong solution. 


\subsection{Maximizing expected utility of terminal wealth}

In this section, we study the optimal investment and premium control policies by maximizing the expected utility of the terminal wealth of the insurance portfolio. Research on optimal investment for insurers maximizing the utility of terminal wealth can be found in Browne (1995), Irgens and Paulsen (2004), Yang and Zhang (2005), Bai and Guo (2008), Liang et al. (2012), Liang and Bayraktar (2013), and references therein.

Assume that the insurer's wealth utility function, denoted by $U(x)$, is a strictly increasing and concave function, i.e., $U^{\prime}(x)>0$ and $U^{\prime \prime}(x)<0$. Associated with each pair of policy $(\pi, u) \in \mathcal{Z}$, the performance function is defined as

$$
V^{\pi, u}(t, x)=\mathbb{E}\left[U\left(Y_{T}^{\pi, u}\right) \mid Y_{t}^{\pi, u}=x\right],
$$

and the value function is defined as

$$
V(t, x)=\sup _{(\pi, u) \in \mathcal{Z}} V^{\pi, u}(t, x) .
$$

Theorem 3.1. The value function $V(t, x)$ of (3.5) is strictly increasing and concave with respect to $x$.

Proof. Given any initial surplus $x$ and a pair of policy $(\pi, u) \in \mathcal{Z}$, the controlled surplus process, say $\left\{X_{t}^{\pi, u}\right\}$, is determined by (3.1) with $X_{t}^{\pi, u}=x$. On the other hand, for any initial surplus $y>x$, if we take the same pair of policy $(\pi, u)$, then the controlled surplus process $\left\{Y_{t}^{\pi, u}\right\}$ is also determined by (3.1) with $Y_{t}^{\pi, u}=y$. It is clear that $d\left(Y_{s}^{\pi, u}-X_{s}^{\pi, u}\right)=0$ for any $t<s<T$, and hence

$$
Y_{T}^{\pi, u}-X_{T}^{\pi, u}=Y_{t}^{\pi, u}-X_{t}^{\pi, u}=y-x>0 .
$$

Note that the insurer's wealth utility function $U(x)$ is strictly increasing with respect to $x$. It follows that

$$
V^{\pi, u}(x, t)=\mathbb{E}\left[U\left(X_{T}^{\pi, u}\right) \mid X_{t}^{\pi, u}=x\right]<\mathbb{E}\left[U\left(Y_{T}^{\pi, u}\right) \mid Y_{t}^{\pi, u}=y\right]
$$

Taking supremum on both sides of the above inequality yields

$$
V(x, t)<\sup _{(\pi, u) \in \mathcal{Z}} \mathbb{E}\left[U\left(Y_{T}^{\pi, u}\right) \mid Y_{t}^{\pi, u}=y\right] \leq V(y, t)
$$


which implies that $V(x, t)$ is a strictly increasing function with respect to $x$.

In addition, for any given initial values $x_{1}$ and $x_{2}$, we take any two pairs of policies $\left(\pi_{1}, u_{1}\right) \in \mathcal{Z}$ and $\left(\pi_{2}, u_{2}\right) \in \mathcal{Z}$, respectively. Let $0 \leq p \leq 1$. Then, for the initial value $p x_{1}+(1-p) x_{2}$, we construct a pair of policy $(\bar{\pi}, \bar{u})$ such that $\bar{\pi}=p \pi_{1}+(1-p) \pi_{2}$ and $\bar{u}=p u_{1}+(1-p) u_{2}$. It is easy to verify that $(\bar{\pi}, \bar{u}) \in \mathcal{Z}$. Furthermore, it follows from (3.1) that

$$
\begin{aligned}
& d\left(p Y_{t}^{\pi_{1}, u_{1}}+(1-p) Y_{t}^{\pi_{2}, u_{2}}\right) \\
= & {\left[r\left(p Y_{t}^{\pi_{1}, u_{1}}+(1-p) Y_{t}^{\pi_{2}, u_{2}}\right)+\mu\left(p G\left(u_{1 t}\right)+(1-p) G\left(u_{1 t}\right)\right)+\bar{\pi}_{t}(\alpha-r)-c\right] d t } \\
& +\sigma \bar{u}_{t} d W_{t}^{Y}+\beta \bar{\pi}_{t} d W_{t}^{S} \\
\leq & {\left[r Y_{t}^{\bar{\pi}, \bar{u}}+\mu G\left(\bar{u}_{t}\right)+\bar{\pi}_{t}(\alpha-r)-c\right] d t+\sigma \bar{u}_{t} d W_{t}^{Y}+\beta \bar{\pi}_{t} d W_{t}^{S} } \\
= & d Y_{t}^{\bar{\pi}, \bar{u}},
\end{aligned}
$$

where the inequality follows from the concavity of $G$ and the comparison theorem of stochastic differential equation (See Karatzas and Shreve (1988)). This inequality implies that, for any $t \geq 0$,

$$
p Y_{t}^{\pi_{1}, u_{1}}+(1-p) Y_{t}^{\pi_{2}, u_{2}} \leq Y_{t}^{\bar{\pi}, \bar{u}}
$$

Given the events $\left\{Y_{t}^{\pi_{1}, u_{1}}=x_{1}\right\},\left\{Y_{t}^{\pi_{2}, u_{2}}=x_{2}\right\}$ and $\left\{Y_{t}^{\bar{\pi}, \bar{u}}=p x_{1}+(1-p) x_{2}\right\}$, by the increasing property and the concavity of the utility function $U$, we obtain

$$
\begin{aligned}
& p V^{\pi_{1}, u_{1}}\left(t, x_{1}\right)+(1-p) V^{\pi_{2}, u_{2}}\left(t, x_{2}\right) \\
= & p \mathbb{E}\left[U\left(Y_{T}^{\pi_{1}, u_{1}}\right) \mid Y_{t}^{\pi_{1}, u_{1}}=x_{1}\right]+(1-p) \mathbb{E}\left[U\left(Y_{T}^{\pi_{2}, u_{2}}\right) \mid Y_{t}^{\pi_{2}, u_{2}}=x_{2}\right] \\
\leq & \mathbb{E}\left[U\left(p Y_{T}^{\pi_{1}, u_{1}}+(1-p) Y_{T}^{\pi_{2}, u_{2}}\right) \mid p Y_{t}^{\pi_{1}, u_{1}}+(1-p) Y_{t}^{\pi_{2}, u_{2}}=p x_{1}+(1-p) x_{2}\right] \\
\leq & \mathbb{E}\left[U\left(Y_{T}^{\bar{\pi}, \bar{u}}\right) \mid Y_{t}^{\bar{\pi}, \bar{u}}=p x_{1}+(1-p) x_{2}\right] .
\end{aligned}
$$

Then, taking supremum on both sides of the above inequality, we have

$$
p V\left(t, x_{1}\right)+(1-p) V\left(t, x_{2}\right) \leq V\left(t, p x_{1}+(1-p) x_{2}\right)
$$

which implies the concavity of $V(t, x)$ with respect to $x$.

To study the optimization problem, we use the techniques of dynamic programming principle. If the value function $V(t, x) \in \mathcal{C}^{1,2}$, then one can apply the standard procedure of 
dynamic programming principle (see Fleming and Soner (2006)) to show that $V(t, x)$ satisfies the Hamilton-Jacobi-Bellman (HJB) equation

$$
\sup _{(\pi, u) \in \mathcal{Z}} \mathcal{A}^{\pi, u} V(t, x)=0
$$

with boundary condition $V(T, x)=U(x)$, where the generator $\mathcal{A}^{\pi, u}$ is defined in (3.2). The following verification theorem tells us that if we can find a solution to (3.6) under suitable conditions, then the solution coincides with the value function.

Theorem 3.2. Suppose that $w(t, x) \in \mathcal{C}^{1,2}$ is a strictly increasing and concave (with respect to $x$ ) solution to HJB equation (3.6) with boundary condition $w(T, x)=U(x)$. Then, $w(t, x)$ is the value function, i.e.,

$$
V(t, x)=w(t, x), \quad t \leq T
$$

Proof. Give any pair of policy $(\pi, u) \in \mathcal{Z}$, since $w(t, x)$ is a $\mathcal{C}^{1,2}$ function, we apply Itô's formula to $w\left(t, Y_{t}^{\pi, u}\right)$. It follows that for $0 \leq t \leq s \leq T$

$$
\begin{aligned}
w\left(s, Y_{s}^{\pi, u}\right) & =w\left(t, Y_{t}^{\pi, u}\right)+\int_{t}^{s} \mathcal{A}^{\pi, u} w\left(v, Y_{v}^{\pi, u}\right) d v+\int_{t}^{s} \sigma u_{v} d W_{v}^{Y}+\int_{t}^{s} \beta \pi_{v} d W_{v}^{S} \\
& \leq w\left(t, Y_{t}^{\pi, u}\right)+\int_{t}^{s} \sigma u_{v} d W_{v}^{Y}+\int_{t}^{s} \beta \pi_{v} d W_{v}^{S},
\end{aligned}
$$

where the last step is due to the fact that $w(t, x)$ is a solution to (3.6). It follows from the boundedness of $u$ and Condition (3.3) for $\pi$ that the last two terms of (3.7) are martingales. Taking conditional expectation and letting $s=T$, we have

$$
w(t, x) \geq \mathbb{E}\left[w\left(T, Y_{T}^{\pi, u}\right) \mid Y_{t}^{\pi, u}=x\right]=\mathbb{E}\left[U\left(Y_{T}^{\pi, u}\right) \mid Y_{t}^{\pi, u}=x\right]
$$

which implies that

$$
w(t, x) \geq \sup _{(\pi, u) \in \mathcal{Z}} \mathbb{E}\left[U\left(Y_{T}^{\pi, u}\right) \mid Y_{t}^{\pi, u}=x\right]=V(t, x)
$$

On the other hand, since $w(t, x)$ is a solution to

$$
w_{t}+\sup _{(\pi, u) \in \mathcal{Z}}\left\{\frac{1}{2}\left[\sigma^{2} u^{2}+\beta^{2} \pi^{2}\right] w_{x x}+[r x-c+\mu G(u)+\pi(\alpha-r)] w_{x}\right\}=0
$$


we can use standard calculus to obtain the maximizers

$$
\begin{aligned}
\pi^{*}(t, x) & =-\frac{\alpha-r}{\beta^{2}}\left(\frac{w_{x}}{w_{x x}}\right), \\
\frac{u^{*}(t, x)}{G^{\prime}\left(u^{*}(t, x)\right)} & =-\frac{\mu}{\sigma^{2}}\left(\frac{w_{x}}{w_{x x}}\right) .
\end{aligned}
$$

Note that $w_{x}>0, w_{x x}<0$, and $G(x)$ is a concave function. We see from Lemma 2.1 that $x / G^{\prime}(x) \rightarrow 0$ as $x \rightarrow 0+$ and $x / G^{\prime}(x) \rightarrow \infty$ as $x \rightarrow \bar{u}-$. Also,

$$
\left(\frac{x}{G^{\prime}(x)}\right)^{\prime}=\frac{G^{\prime}(x)-x G^{\prime \prime}(x)}{G^{\prime 2}(x)}>0, \quad 0<x<\bar{u} .
$$

All these imply that (3.11) uniquely determines $u^{*}(t, x) \in[0, \bar{u}]$. Let $\pi^{*}=\left\{\pi^{*}\left(t, Y_{t}^{\pi^{*}, u^{*}}\right), t \geq\right.$ $0\}$ and $u^{*}=\left\{u^{*}\left(t, Y_{t}^{\pi^{*}, u^{*}}\right), t \geq 0\right\}$. It follows that $\left(\pi^{*}, u^{*}\right) \in \mathcal{Z}$. If we take the pair of policy $\left(\pi^{*}, u^{*}\right)$, then inequalities in (3.7) and (3.8) become equalities. So, we have $w(t, x)=$ $V^{\pi^{*}, u^{*}}(t, x) \leq V(t, x)$.

Suppose now that the insurer has an exponential utility function

$$
U(x)=k-\frac{\gamma}{\theta} e^{-\theta x}, \quad \gamma>0, \theta>0,
$$

where $\theta$ is the so-called constant absolute risk aversion (CARA) coefficient and (3.12) is the so-called CARA utility function. In this case, we have the following result.

Theorem 3.3. Suppose that the insurer has a CARA utility function (3.12). The optimal investment policy $\pi^{*}$ can be written as

$$
\pi_{t}^{*}=\frac{\alpha-r}{\theta \beta^{2}} e^{-r(T-t)}, \quad t \leq T
$$

and the optimal premium control policy $u^{*}$ is uniquely determined by the equation

$$
u_{t}^{*}=\frac{\mu}{\theta \sigma^{2}} G^{\prime}\left(u_{t}^{*}\right) e^{-r(T-t)}, \quad t \leq T .
$$

The value function has the form

$$
V(t, x)=k-\frac{\gamma}{\theta} \exp \left\{-\theta x e^{r(T-t)}+g(T-t)\right\}, \quad t \leq T,
$$

where the function $g$ is given by (3.20) below. 
Proof. To solve for the optimal policies, the key step is to find a solution $w(t, x)$ to HJB equation (3.6). Since the insurer's utility function has an exponential form, we try to find a solution of the form

$$
w(t, x)=k-\frac{\gamma}{\theta} \exp \left\{-\theta x e^{r(T-t)}+g(T-t)\right\}
$$

where $g(\cdot)$ is a suitable function. The boundary condition $w(T, x)=U(x)$ implies that $g(0)=0$. Then, after some calculations, we get

$$
\begin{aligned}
w_{t} & =[w-k]\left(\theta r x e^{r(T-t)}-g^{\prime}(T-t)\right), \\
w_{x} & =[w-k]\left(-\theta e^{r(T-t)}\right), \\
w_{x x} & =[w-k]\left(\theta^{2} e^{2 r(T-t)}\right) .
\end{aligned}
$$

Inserting (3.18) and (3.19) into (3.10) and (3.11) yields (3.13) and (3.14), respectively. Then, putting (3.17)-(3.19) back into (3.9), one can show that the function $g$ satisfies

$$
g^{\prime}(T-t)=c \theta e^{r(T-t)}-\frac{1}{2} m^{2}-\mu\left(G\left(u_{t}^{*}\right)-\frac{1}{2} u_{t}^{*} G^{\prime}\left(u_{t}^{*}\right)\right) \theta e^{r(T-t)},
$$

where $m=(\alpha-r) / \beta$ is the market price of risk, also called Sharpe Ratio. By noting $g(0)=0$ and integrating $g^{\prime}(x)$ from 0 to $T-t$, we obtain

$$
g(T-t)=\frac{c \theta}{r}\left(e^{r(T-t)}-1\right)-\frac{1}{2} m^{2}(T-t)-\mu \theta \int_{t}^{T}\left(G\left(u_{s}^{*}\right)-\frac{1}{2} u_{s}^{*} G^{\prime}\left(u_{s}^{*}\right)\right) e^{r(T-s)} d s .
$$

Finally, it is easy to verify that the function $w(t, x)$ obtained using (3.16) and (3.20) is a $\mathcal{C}^{1,2}$ function and is also a solution to (3.6) with $w_{x}>0, w_{x x}<0$, and boundary condition $w(T, x)=U(x)$. Then, it follows from Theorem 3.2 that the value function (3.15) holds.

Remark 3.1. In Theorem 3.3, expressions for the optimal investment and premium control policies are given by (3.13) and (3.14), respectively. The optimal investment policy is the same as the one obtained in Browne (1995) when the correlation coefficient of the two Brownian motions is zero. A new feature of the optimization problem studied here is that the optimal investment and premium control policies depends on each other in the way that

$$
\pi_{t}^{*}=\frac{(\alpha-r)}{\beta^{2}} \cdot \frac{\sigma^{2}}{\mu} \cdot \frac{u_{t}^{*}}{G^{\prime}\left(u_{t}^{*}\right)},
$$


which can be obtained from either (3.10) and (3.11), or (3.13) and (3.14). We also see that the optimal investment does not depend on the insurance model but depends on the parameters $\alpha$ and $\beta$ of the risky asset, and that the optimal premium control does not depend on the risky asset but depends on the parameters $\mu$ and $\sigma$ of the insurance model and the function $G$. These findings are likely due to the assumption that the two Brownian motions of the risky asset and the insurance model are independent of each other.

\subsection{Minimizing probability of ruin}

In this subsection, we consider the optimization objective of minimizing the ruin probability of the insurance portfolio. Recent research on the optimal investment for insurers minimizing the probability of ruin can be found in Hipp and Plum (2000, 2003), Schmidli (2002), Liu and Yang (2004), Promislow and Young (2005), Luo et al. (2008), and references therein.

Given any pair of policy $(\pi, u) \in \mathcal{Z}$, the insurance portfolio's surplus process $\left\{Y_{t}^{\pi, u}\right\}$ is still governed by (3.1). We define the first time that $\left\{Y_{t}^{\pi, u}\right\}$ hits the level $z$ as

$$
\tau_{z}^{\pi, u}=\inf \left\{t \geq 0 ; Y_{t}^{\pi, u}=z\right\}
$$

Then, the performance function is defined as

$$
\psi^{\pi, u}(x)=\mathbb{P}\left\{\tau^{\pi, u}<\infty \mid Y_{0}^{\pi, u}=x\right\},
$$

and the value function is defined as

$$
\psi(x)=\inf _{(\pi, u) \in \mathcal{Z}} \psi^{\pi, u}(x)
$$

The objective of this section is to derive explicit expression for the value function $\psi(x)$ and find optimal investment and premium control policies $\left(\pi^{*}, u^{*}\right) \in \mathcal{Z}$ such that $\psi(x)=$ $\psi^{\pi^{*}, u^{*}}(x)$.

For the current wealth level $x \geq c / r$, if we choose a special policy with $\pi=0$ and $u=0$, then the volatility of the controlled surplus process $\left\{Y_{t}^{\pi, u}\right\}$ is zero but the drift $r x-c \geq 0$. Under this pair of policy, the probability of ruin is 0 . Thus, we can conclude that $\psi(x)=0$ for all $x \geq c / r$. 
We now investigate the value function $\psi(x)$ for $0 \leq x<c / r$. Suppose that the value function $\psi$ of (3.21) is a $\mathcal{C}^{2}$ function. By the standard procedure of dynamic programming principle again, we can show that $\psi$ satisfies the HJB equation

$$
\inf _{(\pi, u) \in \mathcal{Z}} \mathcal{A}^{\pi, u} \psi(x)=0 .
$$

Since $\psi$ is independent of time $t$, the HJB equation is equivalent to

$$
\inf _{(\pi, u) \in \mathcal{Z}}\left\{\frac{1}{2}\left(\sigma^{2} u^{2}+\beta^{2} \pi^{2}\right) \psi^{\prime \prime}(x)+(\mu G(u)+(\alpha-r) \pi+r x-c) \psi^{\prime}(x)\right\}=0,
$$

for $0 \leq x \leq c / r$. Also, the value function $\psi$ satisfies the boundary conditions $\psi(0)=1$ and $\psi(c / r)=0$.

Theorem 3.4. Suppose that the value function $\psi$ of (3.21) is a $\mathcal{C}^{2}$ function. Then, $\psi$ is a strictly decreasing and convex function on $(0, c / r)$.

Proof. Under the assumption of the theorem, $\psi$ is a continuous function. Given any $c / r>$ $x>y>0$, by the dynamic programming principle, we have

$$
\psi(x)=\inf _{(\pi, u) \in \mathcal{Z}} \mathbb{P}^{x}\left\{\tau_{y}^{\pi, u}<\infty\right\} \psi(y)
$$

Taking a special policy with $\pi=c /(\alpha-\mu)$ and $u=0$ yields $\mathbb{P}^{x}\left\{\tau_{y}^{\pi, u}<\infty\right\}<1$. So, $\psi(x)<\psi(y)<1$ for any $c / r>x>y>0$.

To show the convexity of the value function $\psi(x)$, the method used in Theorem 3.1 does not work anymore. It is because that the ruin probability is the expectation of an indicator function, but the indicator function is not a concave or convex function. Fortunately, with the assumption that $\psi$ is a $\mathcal{C}^{2}$ function, the convexity of the value function $\psi$ can be shown using HJB equation (3.22). The proof of the convexity is similar to the one given in Schmidli (2002). Assume that there exists a point $x \in(0, c / r)$ such that $\psi^{\prime \prime}(x)<0$. Note that $G(u)$ is bounded for $u \in[0, \bar{u}]$. Then, the infimum of the left-hand side of (3.22) tends to $-\infty$ rather than 0 . Thus, we have $\psi^{\prime \prime}(x) \geq 0$. In addition, if there exists a point $x \in(0, c / r)$ such that $\psi^{\prime \prime}(x)=0$, then (3.22) follows only if $\psi^{\prime}(x)=0$. However, this contradicts with the strictly decreasing property of $\psi$ on $(0, c / r)$. Thus, we can conclude that $\psi^{\prime \prime}(x)>0$ for all $0<x<c / r$. 
We next present the verification theorem.

Theorem 3.5. Suppose that $v(x) \in \mathcal{C}^{2}$ on $(0, c / r)$ is a strictly decreasing and convex solution to HJB equation (3.22) with boundary conditions $v(0)=1$ and $v(c / r)=0$. Then, $v(x)$ is the value function, i.e.,

$$
\psi(x)=v(x), \quad 0 \leq x \leq \frac{c}{r} .
$$

Proof. We omit the proof as it is similar to that of Theorem 3.2.

Given a pair of policy $(\pi, u) \in \mathcal{Z}$ such that $\pi$ and $u$ are Markov control policy (feedback policy). That is, both $\pi_{t}$ and $u_{t}$ are functions of the controlled state $Y_{t}^{\pi, u}$, i.e., $\pi_{t}=\pi\left(Y_{t}^{\pi, u}\right)$ and $u_{t}=u\left(Y_{t}^{\pi, u}\right)$. Then, the controlled surplus process can be expressed as

$$
d Y_{t}^{\pi, u}=\Upsilon\left(Y_{t}^{\pi, u}\right) d t+\sigma u\left(Y_{t}^{\pi, u}\right) d W_{t}^{Y}+\beta \pi\left(Y_{t}^{\pi, u}\right) d W_{t}^{S},
$$

where

$$
\Upsilon(x)=r x-c+\mu G(u(x))+(\alpha-r) \pi(x) .
$$

Also, the scale function of (3.23) is given by

$$
S(x)=\int_{0}^{x} \exp \left\{-\int_{0}^{t} \frac{2 \Upsilon(x)}{\sigma^{2} u^{2}(s)+\beta^{2} \pi^{2}(x)} d s\right\} d t,
$$

which can be used to express the value function.

In the following theorem, we derive the optimal investment and premium control policies which minimize the probability of ruin.

Theorem 3.6. For minimizing the ruin probability, the optimal premium control policy $0 \leq u^{*}(x)<\bar{u}$ is uniquely determined by the equation

$$
\frac{1}{2}\left(\frac{m \sigma}{\mu}\right)^{2} \frac{u}{G^{\prime}(u)}+G(u)-\frac{1}{2} u G^{\prime}(u)=\frac{c-r x}{\mu}, \quad 0 \leq x \leq c / r
$$

such that $u^{*}(c / r)=0$, and the optimal investment policy $\pi^{*}(x)$ is given by

$$
\pi^{*}(x)=\frac{(\alpha-r)}{\beta^{2}} \cdot \frac{\sigma^{2}}{\mu} \cdot \frac{u^{*}(x)}{G^{\prime}\left(u^{*}(x)\right)},
$$

such that $\pi^{*}(c / r)=0$. Then, the value function is given by

$$
\psi(x)=\psi^{\pi^{*}, u^{*}}(x)=1-\frac{S^{*}(x)}{S^{*}(c / r)}, \quad 0 \leq x \leq c / r,
$$

where $S^{*}$ is defined in (3.24) with $\pi(x)$ and $u(x)$ replaced by $\pi^{*}(x)$ and $u^{*}(x)$, respectively. 
Proof. Note that the value function $\psi$ is shown to be strictly decreasing and convex in Theorem 3.4. According to the verification theorem, we need to find a strictly decreasing and convex solution, say $v$, to (3.22). Clearly, the infimum in (3.22) with respect to $\pi$ and $u$ exists due to the strict convexity of $\psi$. Taking derivatives with respect to $\pi$ and $u$, we obtain the minimizers satisfying

$$
\begin{aligned}
\pi^{*} & =-\frac{\alpha-r}{\beta^{2}}\left(\frac{v_{x}}{v_{x x}}\right), \\
u^{*} & =-\frac{\mu G^{\prime}\left(u^{*}\right)}{\sigma^{2}}\left(\frac{v_{x}}{v_{x x}}\right),
\end{aligned}
$$

which implies a relationship between $u^{*}$ and $\pi^{*}$, i.e., (3.26). On the other hand, if we put (3.27) and (3.28) back into (3.22), then the HJB equation turns out to be

$$
\left[r x-c+\frac{1}{2}(\alpha-r) \pi^{*}+\mu\left(G\left(u^{*}\right)-\frac{1}{2} u^{*} G^{\prime}\left(u^{*}\right)\right)\right] v_{x}=0,
$$

which implies, by the strictly decreasing property of $v$, that

$$
r x-c+\frac{1}{2}(\alpha-r) \pi^{*}+\mu\left(G\left(u^{*}\right)-\frac{1}{2} u G^{\prime}\left(u^{*}\right)\right)=0 .
$$

Combining (3.26) with (3.30), we see that $u^{*}=u^{*}(x)$ is a solution to (3.25). Note that $G(0)=0, G^{\prime}(\bar{u})=0, u G^{\prime}(u) \rightarrow 0$, and $u / G^{\prime}(u) \rightarrow 0$ as $u \rightarrow 0+$ by Lemma 2.1. Thus, the left-hand side of (3.25) equals 0 as $u \rightarrow 0+$ and $\infty$ as $u \rightarrow \bar{u}$. In addition, taking derivative with respect to $u$ on the left-hand side of (3.25) yields

$$
\frac{1}{2}\left(G^{\prime}(u)-u G^{\prime \prime}(u)\right)\left(1+\left(\frac{m \sigma / \mu}{G^{\prime}(u)}\right)^{2}\right)>0,
$$

for $0<u<\bar{u}$ because $G$ is strictly increasing and concave on $(0, \bar{u})$. Thus, for $0<x<c / r$, the optimal policy $0 \leq u^{*}(x)<\bar{u}$ is uniquely determined by (3.25) such that $u^{*}(c / r)=0$, and the optimal investment policy $\pi^{*}=\pi^{*}(x)$ is determined by (3.26). Furthermore, it follows from $u^{*}(c / r)=0$ and (2.6) in Lemma 2.1 that $\pi^{*}(c / r)=0$.

Finally, it is easy to verify that $u^{*}$ and $\pi^{*}$ obtained in (3.25) and (3.26) respectively belong to $\mathcal{Z}$. Hence, the solution $v$ to HJB equation (3.22) is a solution to

$$
\frac{1}{2}\left(\sigma^{2} u^{* 2}(x)+\beta^{2} \pi^{* 2}(x)\right) v^{\prime \prime}(x)+\left(\mu G\left(u^{*}(x)\right)+(\alpha-r) \pi^{*}(x)+r x-c\right) v^{\prime}(x)=0,
$$


with boundary conditions $v(0)=1$ and $v(c / r)=0$. Solving this yields

$$
v(x)=\psi^{\pi^{*}, u^{*}}(x)=1-\frac{S^{*}(x)}{S^{*}(c / r)}, \quad 0 \leq x \leq \frac{c}{r} .
$$

Then, the result follows from the verification theorem (Theorem 3.5).

Remark 3.2. From the above analysis, we know that the left-hand side of (3.25) is strictly increasing with respect to $u$. Thus, the solution to (3.25), $u^{*}(x)$, is strictly decreasing with respect to $x$. In addition, since the function $u / G^{\prime}(u)$ is a strictly increasing function of $u$, we see from (3.26) that $\pi^{*}(x)$ is strictly increasing with respect to $u^{*}(x)$, and hence is strictly decreasing with respect to $x$. Furthermore, for the special case with $m=0$ (i.e., $\alpha=r$ ), it follows from (3.26) that the optimal investment policy $\pi^{*}=0$, and follows from (3.25) that the optimal premium control policy $u^{*}$ is the solution to

$$
G(u)-\frac{1}{2} u G^{\prime}(u)=\frac{c-r x}{\mu},
$$

which is independent of $\sigma^{2}$, the second moment of the claim sizes.

Remark 3.3. In the paper of Browne (1995), it is shown that in the absence of risk-free asset in the market (i.e., $r=0$ ), the optimal investment policy for the maximization of exponential utility of terminal wealth and the minimization of ruin probability are equivalent by choosing suitable risk aversion coefficient, but this does not hold for $r>0$. In this paper, this result also holds by observing (3.13) and (3.26). Let the constant $u^{*}$ be the solution to (3.25) when $r=0$. Then we can choose

$$
\theta=\frac{\mu}{\sigma^{2}} \cdot \frac{G^{\prime}\left(u^{*}\right)}{u^{*}}
$$

which implies that the optimal investment policies under the two criteria are equivalent.

Remark 3.4. A special policy with $\pi=0$ and $u=0$ is optimal, and the corresponding ruin probability satisfies $\psi(x)=0$ for all $x \geq c / r$. This fact implies that when minimizing ruin probability, the company will withdraw from the market when its wealth level exceeds the level $c / r$. However, this is not the case at all in practice. From this point of view, the criterion of minimizing ruin probability is somehow conservative, especially for large insurance companies. 


\section{An Example}

In Section 3, we obtain the optimal investment and premium control policies under the two criteria: maximizing the expected utility of terminal wealth and minimizing the probability of ruin. For the applications of our model in practice, the functional form of $G$ should be estimated from the empirical data of an insurance portfolio, or specified based on the industry experience. In this section, we give a specific form of the function $G$ to show that model (2.5) represents a class of non-linear stochastic control risk model.

Let

$$
G(u)=u\left(\sqrt{\lambda}_{\max }-u\right), \quad 0 \leq u \leq \sqrt{\lambda}_{\max },
$$

from which we have

$$
\lambda_{t}=h\left(\theta_{t}\right)=\frac{\lambda_{\max }}{1+\theta_{t}^{2}}
$$

Clearly, the function $G$ is a strictly concave function such that $G(0)=G\left(\sqrt{\lambda}_{\text {max }}\right)=0$. It is also easy to verify that it satisfies properties (2.6) in Lemma 2.1. Then, with the function (4.1), the diffusion model of the insurance portfolio without investment turns out to be

$$
d Y_{t}=\left(b u_{t}-a u_{t}^{2}-c\right) d t+\sigma u_{t} d W_{t}^{Y}
$$

where $a=\mu, b=\mu \sqrt{\lambda}_{\text {max }}$, and $c$ are positive parameters. As a special case of our model of study (2.5), model (4.2) has been extensively studied in recent years. For example, Guo et al. (2004) study the singular optimal dividend control problem; Meng et al. (2013) extend the study of optimal dividends to the case with impulse dividend policy; and Zhou and Yuen (2012) examine an optimal reinsurance and dividend problem under the variance premium principle, which also leads to an optimization problem for model (4.2).

In this section, we set $u$ as a premium control variable and study the optimal investment policy and premium control policy for model (4.2). For the criterion of maximizing the expected utility of terminal wealth, if the insurer has a CARA utility, then the optimal investment and premium control policies are determined by (3.13) and (3.14). With the 
function $G$ of (4.1), we have

$$
\begin{aligned}
\pi_{t}^{*} & =\frac{\alpha-r}{\theta \beta^{2}} e^{-r(T-t)}, \\
u_{t}^{*} & =\frac{b}{2 a+\frac{\theta \sigma^{2}}{\mu} e^{r(T-t)}}=\frac{\sqrt{\lambda}_{\max }}{2+\frac{\theta \sigma^{2}}{\mu^{2}} e^{r(T-t)}},
\end{aligned}
$$

for $t \leq T$. As was mentioned in Remark 3.1, the optimal investment policy is the same as the one obtained in Browne (1995). The optimal premium control policy decreases with the insurer's CARA coefficient $\theta$ as well as with the time period $T-t$, and attains the maximum value $\sqrt{\lambda}_{\max } /\left(2+\theta \sigma^{2} / \mu^{2}\right)$ at time $t=T$. For the criterion of minimizing the probability of ruin, by substituting the function $G$ of (4.1) into (3.25) and (3.26), one can show that $u^{*}(x)$ is the root of

$$
2 \mu b u^{2}-\left[b^{2}+(m \sigma)^{2}+4(c-r x) \mu\right] u+2(c-r x) b=0,
$$

with $b=\mu \sqrt{\lambda}_{\max }$. Since $\bar{u}=b / 2 a=\sqrt{\lambda}_{\max } / 2$, we need to find the root $u^{*}(x)$ such that $0<u^{*}(x)<\bar{u}$. Thus, we obtain the optimal premium control policy

$$
u^{*}(x)=\frac{b^{2}+(m \sigma)^{2}+4(c-r x) \mu-\sqrt{(m \sigma)^{4}+\left(b^{2}-4(c-r x) \mu\right)^{2}+2\left(b^{2}+4(c-r x)\right)(m \sigma)^{2}}}{4 \mu b},
$$

and the optimal investment policy

$$
\pi^{*}(x)=\frac{(\alpha-r)}{\beta^{2}} \cdot \frac{\sigma^{2}}{\mu} \cdot \frac{u^{*}(x)}{b-2 \mu u^{*}(x)} .
$$

In particular, for $m=0$ (i.e., $\alpha=r$ ), we have $\pi^{*}(x)=0$, which means that we invest all the surplus in the risk-free asset. In this case, the optimal premium control policy becomes

$$
u^{*}(x)=\frac{b^{2}+4(c-r x)-\sqrt{\left(b^{2}-4(c-r x)\right)^{2}}}{4 \mu b}=\frac{2(c-r x)}{\mu b},
$$

where the last equality follows from Assumption $\mathrm{C}$ as $G(\bar{u})>c / \mu$ implies that $b^{2}>4 c>$ $4(c-r x)$ for $0<x<c / r$.

\section{Concluding remarks}

Inspired by the previous studies on optimal premium rate, we introduce a link between the safety loading and claim arrival rate of an insurance portfolio. Based on the link function, we 
then propose an nonlinear diffusion model in which the premium rate and the investment can be served as control variables. This nonlinear diffusion model is obtained by investigating the diffusion approximation to a non-homogeneous compound Poisson process. The optimal investment and premium control policies and the corresponding value functions are explicitly obtained under the two criteria: maximizing exponential utility of terminal wealth and minimizing ruin probability. For the applications of our model in practice, the functional form of $G$ in model (2.5) should be estimated from the empirical data of an insurance portfolio, or specified based on the industry experience. To see the application of the nonlinear model, we present an example for a specific form of the link function between the safety loading and claim arrival rate. In this special case, the model reduces to a quadratic diffusion model studied by Guo et al. (2004). This example shows that our proposed model represents a class of non-linear stochastic control risk model. As a by-product, we find another way to describe the well-known quadratic diffusion model.

\section{Acknowledgements}

The research of Ming Zhou The research of Kam Chuen Yuen was supported by a grant from the Research Grants Council of the Hong Kong Special Administrative Region, China (Project No. HKU17329216). The research of Chuancun Yin

\section{References}

[1] Asmussen, S., Christensen, B. J. and Taksar, M. 2013. Portfolio size as function of the premium: modelling and optimization. Stochastics 85(4): 575-588.

[2] Bai, L. and Guo, J. 2008. Optimal proportional reinsurance and investment with multiple risky assets and no-shorting constraint. Insurance: Mathematics and Economics, 42: 968-975. 
[3] Browne, S. 1995. Optimal investment policies for a firm with a random risk process: exponential utility and minimizing the probability of ruin. Mathematics of Operations Research 20: 937-958.

[4] Fleming, W.H. and Soner, M. 2006. Controlled Markov Processes and Viscosity Solutions, 2nd edition. Springer, New York.

[5] Guo, X., Liu, J. and Zhou, X. Y. 2004. A constrained non-linear regular-singular stochastic control problem, with applications. Stochastic Processes and their Applications 109: $167-187$.

[6] Hipp, C. and Plum, M. 2000. Optimal investment for insurers. Insurance: Mathematics and Economics 27: 215-228.

[7] Hipp, C. and Plum, M. 2003. Optimal investment for investors with state dependent income, and for insurers. Finance and Stochastics 7: 299-321.

[8] Højgaard, B. 2002. Optimal dynamic premium control in non-life insurance. Maximizing dividend pay-outs. Scandinavian Actuarial Journal: 225-245.

[9] Højgaard, B. and Taksar, M. 2001. Optimal risk control for a large corporation in the presence of returns on investments. Finance and Stochastics 5: 527-547.

[10] Irgens, C. and Paulsen, J. 2004. Optimal control of risk exposure, reinsurance and investments for insurance portfolios. Insurance: Mathematics and Economics 35: 2151.

[11] Jabari, S. E. and Liu, X. 2013. A stochastic model of traffic flow: Gaussian approximation and estimation. Transportation Research Part B 47: 15-41.

[12] Karatzas, I. and Shreve, S.E. 1988. Brownian Motion and Stochastic Calculus. SpringerVerlag, Berlin.

[13] Liang, Z., Yuen, K. C and Guo, J. 2011. Optimal proportional reinsurance and investment in a stock market with Ornstein-Uhlenbeck process. Insurance: Mathematics and Economics 49: 207-215. 
[14] Liang, Z. and Bayraktar, E. 2014. Optimal reinsurance and investment with unobservable claim size and intensity. Insurance: Mathematics and Economics 55: 156-166.

[15] Liu, C. and Yang, H. 2004. Optimal investment for an insurer to minimize its probability of ruin. North American Acruarial Journal 8: 11-31.

[16] Luo, S., Taksar, M. and Tsoi, A. 2008. On reinsurance and investment for large insurance portfolios. Insurance: Mathematics and Economics 42: 434-444.

[17] Martin-Löf. 1983. Premium control in an insurance system, an approach using linear control theory. Scandinavian Actuarial Journal: 1-27.

[18] Meng, H., Siu, T. K. and Yang, H. 2013. Optimal dividends with debts and nonlinear insurance risk processes. Insurance: Mathematics and Economics, 53: 110-121.

[19] Promislow, S. D. and Young, V.R. 2005. Minimizing the probability of ruin when claims follow Brownian motion with drift. The North American Actuarial Journal, 9(3): 109128.

[20] Schmidli, H. 2008. Stochastic Control in Insurance. Springer-Verlag, London.

[21] Schmidli, H. 2002. On minimizing the ruin probability by investment and reinsurance. The Annals of Applied Probability 12: 890-907.

[22] Vandebroeka, M. and Dhaenea, J. 1990. Optimal premium control in a non-life insurance business. Scandinavian Actuarial Journal: 3-13.

[23] Yang, H. and Zhang, L. 2005. Optimal investment for insurer with jump-diffusion risk process. Insurance: Mathematics and Economics 37: 615-634.

[24] Zhou, M. and Yuen, K.C. 2012. Optimal reinsurance and dividend for a diffusion model with capital injection: Variance premium principle. Economic Modelling 29: 198-207. 